Z. klin. Chem. u. klin. Biochem.

7. Jg., S. $83-88$, Januar 1969

\title{
Analyse Automatique Continue, Discontinue et Analyse Rapide')
}

\author{
Pat J. Bierens de HaAn \\ Laboratoire central Hopital cantonal, 1211 Geneve 4
}

(Eingegangen am 4. November 1968)

\section{Introduction}

La crise que traversent actuellement les laboratoires de chimie clinique n'est pas tant une affaire de main d'oeuvre et de locaux que d'organisation. Les méthodes artisanales, les solutions improvisées et la multiplication du personnel ne suffisent plus à les adapter aux exigences de nos hôpitaux.

Sans doute, cette crise se traduit-elle par un déséquilibre qui va croissant entre le travail de laboratoire et les "écritures", celles-ci se développant exagérément aux dépens de celui-là. Mais on a trop souvent tendance à penser que les ordinateurs contiennent la solution de tous les problèmes du laboratoire central, à surestimer les performances des machines "automatiques" d'analyse, bref à oublier qu'après tout c'est de chimie analytique qu'il s'agit et que c'est par là qu'il faut commencer l'autocritique. Cette attitude conduit à faire traiter par un matériel électronique coûteux des chiffres imprécis voire même faux, parce qu'obtenus à l'aide d'instruments imparfaits. C'est pourquoi nous n'aborderons ici que l'aspect instrumental de la chimie clinique analytique, ou, si l'on préfère, le problème du traitement de la matière, par opposition à celui du traitement de l'information. Et encore ne prétendonsnous pas en faire le tour: les questions économiques qui se posent en termes de prix de revient de l'analyse; compte tenu de l'amortissement des appareils, de la consommation de réactifs, etc. sont éludées. Elles sont primordiales, pour les pays du tiers-monde en particulier, mais il est encore trop tôt pour y répondre.

Enfin, l'accent étant mis sur les dosages de "grande routine", nous serons amenés à énoncer des généralités qui ne concernent naturellement pas certains dosages spéciaux et exceptionnels faisant intervenir des méthodes de séparation ou des temps d'équilibration prolongés.

$\mathrm{Au}$ moment où l'instrumentation passe par une profonde mutation et que l'analyste qui cherche à renouveler son matériel se trouve mis en présence d'une foule de produits disparates, tant par la conception que par les performances et le prix de revient, il est important de dégager des tendances, c'est à dire d'imaginer, d'une part, vers quels types d'appareils concergeront les efforts actuels des fabricants et, d'autre part, de reconnaitre les produits dont la conception est mauvaise et qui, en dépit de certains perfectionnements possibles, sont appelés à disparaittre, à plus ou moins brève échéance. Cette démarche assez négative résulte du manque

1) Vortrag anläßlich der ILMAC, 11. September 1968, Basel. de coopération qui est malheureusement trop souvent la règle entre le fabricant et l'utilisateur (lesquels ne sont d'ailleurs souvent chimistes ni l'un ni l'autre ...).

\section{Analyse Automatique Continue}

Toute réflexion sur l'analyse automatique passe par un examen critique de l'analyse colorimétrique en flux continu. Pendant de nombreuses années les deux choses ont d'ailleurs été presque confondues. Aujourd'hui encore, beaucoup trop d'articles décrivant l'autonıatisation d'une analyse clinique se bornent à introduire une application nouvelle de l'AutoAnalyzer. Quant à la publicité donnée aux fameux symposiums Technicon "Automation in Analytical Chemistry", elle ne vient pas clarifier la situation.

Décrit en 1957 par SkegGs (1), et produit la même année, l'AutoAnalyzer a d'abord été mis au point pour faire face à une surcharge des laboratoires d'analyse clinique, surcharge qui, ainsi que beaucoup de problèmes liés au développement économique, a touché d'abord les Etats-Unis. Limitées tout d'abord à la colorimétrie de l'urée et du glucose sanguins, ses applications se sont bientôt étendues aux principales méthodes d'analyse chimique du sérum et de l'urine, et son emploi généralisé très rapidement, même en Europe où rares sont les laboratoires importants qui n'en possèdent au moins un modèle. ( $\mathrm{Par}$ la suite, l'AutoAnalyzer a gagné l'industrie; il se spécialise de plus en plus dans le contrôle et la mesure de phénomènes continus, comme la chromatographie sur colonne des sucres et des acides aminés, domaine qui est le sien beaucoup plus que l'analyse colorimétrique de routine).

Il est intéressant de remarquer qu'en dix ans l'Auto Analyzer s'est très peu modifié, que ses performances ne se sont guère améliorées et que c'est sous sa forme initiale qu'il est encore presque exclusivement répandu. Les seuls perfectionnements à signaler, au cours de cette période, si on omet l'annexion de la photométrie de flamme et de la fluorimétrie, sont un type de cuvette à flux continu accroissant la sensibilité et un échantillonneur alternatif à réglage souple permettant d'ajuster la cadence et la durée des prélèvements et des intervalles de rinçage.

$\mathrm{Si}$ sa robustesse et sa silhouette, ainsi que son mode d'utilisation nous sont familiers, il n'est pas inutile de rappeler certains aspects fondamentaux de son fonctionnement. C'est ainsi que, plutôt que de considérer l'AutoAnalyzer comme une juxtaposition de modules spécialisés, entre lesquels le mélange de réaction serait 
ingénieusement transporté par des tuyaux, nous préférons y voir d'abord une colonne liquide de 15 à $25 \mathrm{~m}$, segmentéc par des bulles d'air et qui se déplace à une vitesse variable dans un conduit de géométrie complexe, la section, la nature des parois et l'orientation variant dans des proportions considérables. Dans la portion initiale de ce conduit, et alors que la vitesse de la colonne liquide est la plus basse, celle-ci subit sa première modification chimique en recevant, sur une longueur de deux mètres et demi de son trajet, et à travers une paroi poreuse, une certaine quantité de substances, dont celle que l'on cherche à doser. Ces substances proviennent d'un flux parallèle qui se déplace à la même vitesse de l'autre côté de la paroi poreuse. Elles y ont été introduites par injection de sérum.

La concentration moyenne du sérum dans la portion du flux donneur qui l'a reçue pourrait être déterminée approximativement, mais le processus d'échange du flux donneur au flux receveur n'est que partiel, et la concentration des solutés dans ce dernier ne peut être calculée en raison des très nombreux paramètres impliqués. Ce qui signifie en clair que, dès ce stade, on ne sait plus très bien ce qui se passe dans l'AutoAnalyzer. En différents points du conduit stationnaire, d'autres modifications sont apportées à la composition de la colonne liquide mobile, en particulier par l'adjonction de colonnes affluentes renfermant les réactifs, ce qui dilue, accélère le déplacement et modifie la répartition des bulles d'air du flux principal. L'homogénéisation des petits segments liquides individuels est hâtée, en agissant sur la direction de la force gravitationnelle, par une série de circuits circulaires verticaux de faible rayon de courbure, constituant les bobines de mélange. Enfin, le chauffage et le refroidissement de la colonne liquide sont obtenus par thermostatisation de la paroi $\mathrm{du}$ conduit sur des longueurs plus ou moins importantes.

Dans la portion finale du conduit, et alors que la colonne a atteint sa vitesse maximum, une trappe à air la débarrasse de ses bulles et d'une partie du liquide. Il se fait un flux continu, ralenti, sur une dizaine de centimètres. Celui-ci traverse un tube coudé qui joue le rôle de cuvette photométrique, sur quinze millimètres de long environ, et la densité optique, comparée à une valeur fixée arbitrairement, est enregistrée au cours du temps.

Il faut retenir de cette description, d'une part, la tentative pour découper dans l'espace le mélange final en une longue série de portions liquides indépendantes que l'on mesure finalement l'une après l'autre, par une manière de balayage, et, d'autre part, l'existence d'une colonne liquide ininterrompue dont la composition est modifiée par des procédés empiriques, si bien qu'on ne connaît jamais la composition du contenu de la cellule de mesure, ni sa densité optique vraie.

Cette description est encore insuffisante dans la mesure où elle laisse supposer que les segments liquides élémentaires sont réellement séparés par les bulles d'air, alors que leur interaction mutuelle est à l'origine des faiblesses du système. Les gradients de concentration, variables en grandeur et en sens, se traduisent par une diffusion dans l'axe de progression de la colonne. Limitée seulement par la segmentation, elle se fait par le film liquide qui entoure les bulles d'air.

Les irrégularités du parcours, en particulier les modifications brusques de la section du conduit, entraînent une déformation de la colonne ou un freinage momentanné des bulles d'air; il s'ensuit des mouvements de convection qui mélangent le contenu de deux ou plusieurs segments liquides consécutifs.

Le déplacement du liquide le long des parois immobiles du conduit fait apparaître un gradient de vitesse, de la périphérie au centre de la colonne; il s'ensuit des phénomènes de rétention qui dépendent de la viscosité du milieu et de la vitesse de déplacement.

Cet effect, comme celui de l'adsorption des grosses molécules sur les parois de verre, n'agissent que dans le sens opposé à la progression de la colonne liquide. Ils sont responsables de l'asymétrie des pics photométriques, de la formation de queues et de la contamination d'un échantillon par celui qui le précède.

La composition du liquide, en particulier la présence de protéines, la température et la nature des parois en déterminent l'importance. Ces quatre types d'interactions entre les segments sont amplifiées par les dilutions et les accélérations du flux qui leur sont associées et par les passages dans les bobines dé mélange. Elles sont responsables de la forme vaguement gaussienne des signaux enregistrés et de leur interdépendance. Leurs effets ne peuvent être prévus que très approximativement.

Les variations continues de la densité optique du contenu de la cellule de mesure, c'est à dire de sa composition, résultent donc de la superposition de phénomènes d'équilibres lents, interdépendants ou non. La cinétique de cette mesure a été étudiée récemment par Thiers (2) et ses collaborateurs, pour un système très simple puisqu'il ne comportait qu'une colonne liquide constituée alternativement d'eau et de solution colorée rouge. Pour décrire la courbe enregistrée, cet auteur a été amené à introduire une douzaine de termes entièrement nouveaux pour l'analyste, dont un paramètre cinétique, le temps de demi-rinçage, pour évaluer l'importance globale des seuls phénomènes de mélange longitudinal, pour une méthode donnée.

Quant aux conditions hydrodynamiques, elles sont telles que tout ce qui se passe en un point de la colonne liquide affecte l'ensemble du système. En cas de défectuosité ou de panne, ce sont souvent dix essais qui sont altérés ou perdus.

Tout ceci n'empêche pas l'AutoAnalyzer de se porter fort bien, puisque plus de cinquante constituants du sang et de l'urine peuvent être analysés par ce moyen et qu'une part non négligeable de tous les dosages cliniques actuellement exécutés dans le monde se font sur ces dispositifs ... ce qui ne manque pas de déconcerter au premier abord et ne s'explique que par des considérations qui n'ont rien de scientifique, et dont la 
première est que, pour des raisons obscures, l'Auto Analyzer a longtemps été le seul appareil automatique d'analyse disponible.

Si l'on se demande ce dont l'AutoAnalyzer est capable, on s'aperçoit que ses performances ne sont pas très faciles à chiffrer, tant elles dépendent de la manière dont il est utilisé. En moyenne, il exécute quarante dosages à l'heure, parfois soixante, ce qui est un maximum, et parfois vingt, ce qui cst souvent préférable: la qualité des résultats est d'autant meilleure qu'il travaille plus lentement et que la fréquence des échantillons-étalons est plus grande. Et pourtant, la cadence de soixante à l'heurc doit être considérée elle-même comme insuffisante, au vu des exigences actuelles.

La sensibilité des méthodes utilisées est très inférieure à celle des microméthodes manuelles. La quantité de sérum requise ne descend guère au-dessous de $100 \mu$. et se situe plus souvent autour de $300 \mu l$. Ceci s'explique par la dilution initiale de l'échantillon, le rendement médiocre du processus de dialyse, et par l'allongement de la colonne à chaque addition de réactif.

Satisfaisante pour les uns, insuffisante pour d'autres, la précision des résultats est souvent trompeuse, par suite des phénomènes de dérive, communs à toutes les méthodes instrumentales à flux continu. Si, comme le fait Hugries (3), nous distinguons la répétitivité de la reproductibilité, nous dirons que la première est très supéricure à la seconde, c'est à dire qu'un même échantillon dosé dix fois de suite sur le même appareil en l'espace de vingt minutes donnera des résultats superposables à près de $1 \%$, alors que, d'un jour à l'autre ou surtout du début à la fin d'une journée de travail, les écarts observés pourront n'être plus acceptables ... même pour un dosage clinique (il faut réfuter une fois pour toutes ici l'argument selon lequel les médecins se contentent d'une précision moyenne de 5 à $7 \%$ ). Tout le système est en effet soumis à des variations cycliques quotidiennes, auxquelles s'ajoute une évolution progressive due à l'encrassage et à l'usure des éléments élastiques en particulier.

L'exactitude peut être bonne et l'est généralement, mais elle repose entièrement sur la qualité et la fréquence des étalons. Il arrive par conséquent qu'elle échappe au contrôle, surtout lorsque la coloration ne suit pas la loi DE BEER. On ne mesure jamais que des hauteurs de pics, et on peut imaginer le nombre de facteurs impliqués.

Le degré d'automaticité, très poussé, a beaucoup contribué au succès de l'AutoAnalyzer, mais la présentation graphique des résultats requiert une technique de lecture manuelle au cours de laquelle les erreurs s' introduisent facilement.

Il faut donc insister sur le caractère original de la méthode. Le fait qu'elle mette en jeu une réaction colorée ne suffit pas à parler d'automatisation de la colorimétrie. Ainsi, beaucoup de méthodes courantes et largement répandues sur AutoAnalyzer n'ont pas leur équivalent en colorimétrie manuelle classique. La séparation par dialyse permet d'appliquer à la détermination de la créatinine la réaction de JAFFế avec une très grande spécificité, ce qui n'est pas le cas des méthodes manuelles. Le contrôle du temps de réaction par le système continu et l'utilisation des courbes d'étalonnages de forme complexe lors de la lecture, permettent d'appliquer à la détermination de l'urée, la réaction à la diacétylmonoxime, dont on sait: 1) qu'elle donne une coloration instable qui 2) ne suit pas la loi DE BEer.

Le fait que toutes les opérations analytiques se déroulent à l'abri de l'atmosphère, en circuit fermé, permet d'utiliser des mélanges instables, corrosifs, volatils ou d'odeur désagréable beaucoup plus facilement que dans les méthodes manuelles, comme c'est le cas pour le cholestérol, ou le glucose par l'o-toluidine. Il est possible pour la même raison d'appliquer des méthodes de dosage où le produit à déterminer est lui-même volatil, comme pour le $\mathrm{CO}_{2}$ ou l'iode protéique.

L'AutoAnalyzer rend ainsi utilisables des méthodes ou des réactions qui ne le sont pas en chimie analytique classique, on peut s'en féliciter mais il y a là deux dangers dont le premier et le plus grave est qu'une telle pratique va à l'encontre des efforts de normalisation des méthodes de chimie clinique, et risque de faire passer les intérêts des fabricants avant ceux, très différents, des laboratoires de chimie clinique. Le deuxième danger est, au plan scientifique, d'encourager une certaine paresse méthodologique en introduisant l'empirisme jusque dans les réactions chimiques utilisées. (Ce n'est plus de la chimie, c'est de la cuisine! ...) Les nouvelles formes d'automatisation suscitent déjà des travaux très intéressants pour améliorer les déterminations de l'urée (4) et du glucose (5), par exemple, alors que, nous l'avons dit, l'Auto Analyzer n'a pas besoin de ces perfectionnements. Dire ici que seule l'efficacité compte revient à mettre la chimie clinique dans des ornières qui ne peuvent que freiner son développement.

\section{Analyse Automatique Discontinue}

Tout ceci étant le passé et encore le présent, tournonsnous vers l'avenir, vers la belle anarchie qui règne en 1968 dans l'analyse instrumentale mécanisée.

Une constatation s'impose immédiatement, les efforts d'imagination des constructeurs se situent au plan technologique. Tous essayent différemment de faire la même chose, à savoir reproduire le plus fidèlement possible les gestes de la laborantine idéale. Cette humilité est réjouissante pour l'analyste: il pourra adapter l'instrument à la méthode, superposer les résultats manuels aux résultats automatiques; il ne sera plus troublé par des problèmes de physique des fluides; les notions d'addition, de dilution, d'agitation, de lecture photométrique retrouveront leur sens familier.

La plupart de ces nouveaux instruments ont d'autres traits communs: la rapidité, puisqu'ils exécutent cent à trois cents analyses à l'heure, et la sécurité, parce qu'une défaillance ne touche généralement qu'un seul échantillon et qu'en cas de panne, tous les essais en cours 
restent identifiables et utilisables. Tous affichent, impriment ou perforent des résultats immédiatement exploitables.

Dès lors, l'examen critique est grandement facilité, en apparence du moins, puisqu'il suffit de choisir, entre plusieurs instruments qui font la même chose, celui qui la fait le mieux et le plus vite. Malheureusement, les opérations élémentaires impliquées dans une analyse chimique sont assez nombreuses pourqu'aucuninstrument actuellement sur le marché ne soit capable de les effectuer toutes de manière satisfaisante, alors que, et c'est là qu'on peut parler de recherche désordonnée, la plupart des problèmes pris isolément ont trouvé une solution acceptable chez l'ou ou l'autre des constructeurs. Il semblerait donc possible dès maintenant de concevoir une bonne machine, à partir des bons éléments de plusieurs machines médiocres.

La liste du tableau 1 énumère, par ordre alphabétique, quelques-uns des principaux constructeurs qui se sont intéressés à la mécanisation de l'analyse clinique, avec leurs produits. Nous n'avons pas cherché à les classer bien que les dimensions des maisons et les performances des appareils soient d'ordres de grandeurs très différents.

Tableau 1

\begin{tabular}{ll}
\hline \multicolumn{1}{c}{ Firme } & \multicolumn{1}{c}{ Produit } \\
\hline Aga Medical Division (Sweden) & Autochemist \\
Amercan Optical Instr. Co. (USA) & Robot Chemist \\
Baird \& Tatlock (G. B.) & Analmatic \\
Bausch \& Lomb (USA) & Zymat 340 (Spectrozyme) \\
Beckman Instr. Inc. (USA) & DSA-560 \\
B. Braun Melsungen (Germany) & Syste-Matik \\
Beckman Instr = Internat. & Sacas \\
(Genève Glenrothes) & Stuttgarter Automatisches \\
Bühler, Tübingen (Germany) & Analysensystem \\
Du Pont Instr. (USA) & Aca System \\
Elvi-Milano (Italy) & Elvi 300 \\
Eppendorf Geraetebau (Germany) & Eppendorf Analysen System \\
Carlo Erba (Italy) & CLA 1510 \\
Evans Electroselenium Ltd. (G. B.) & Colorimeter-171, Sampler-178 \\
Fisons Ltd. (G. B.) & Dispensing \& Diluting System \\
Gilford Instr. Lab. Inc. (USA) & Microsample Spectrophoto- \\
& meter 300 N \\
Grant \& Linson (Sweden) & Autolab \\
Griffin \& George (G. B.) & Bioanalyst \\
Hycel (USA) & Mark X \\
Instrumenttation Laboratory & Flame Photometer-143, \\
Inc. (USA) & Dilutor-144 \\
Joyce Loebl \& Co. Ltd. (G. B.) & Mecolab \\
LKB Produkter A. B. (Sweden) & Ultrolab System \\
Poli-Mak S. R. L. (Italy) & Clino-Mak, Bio-Mak \\
Quickfit \& Quartz Ltd. (G. B.) & Chematic-617 \\
Satt Medical Elektronics (Sweden) & Automaton \\
Smith Kline Instr. Co. (USA) & Eskalab \\
Struers Scientific Instr. (Denmark) & Samplomat \\
Technicon Instr. Corp. (USA) & SMA 12/30, SMA 12/60 \\
Arthur H. Thomas Co. (USA) & Spectrophotometer Acces- \\
Unicam Instr. Ltd. & sories \\
Vickers Ltd. Medical Group & SP 3000 \\
Carl Zeiss-Oberkochen (Germany) & Multichannel 300 \\
& P4 System \\
\hline & \\
\hline
\end{tabular}

Parmi ces appareils, certains sont fabriqués en série depuis quelques mois ou quelques années, d'autres sont encore à l'état de prototypes, ou sont mis en circulation module par module. D'autres enfin, comme les matériels de Beckman et de DuPont, bien qu'opérationnels sont encore peu connus, ayant été présentés pour la première fois le 18 août dernier à Washington ${ }^{2}$ ). La liste n'est pas exhaustive, car certaines recherches sont encore trop peu avancées pour que le constructeur accepte de les divulguer ou qu'il ait même été désigné. D'autre part, une foule d'appareils de la famille des diluteurs volumétriques et de celle des échantillonneurs photométriques en particulier ne figurent pas ici pour des raisons de place.

Nous avons énuméré sur le tableau 2 les opérations élémentaires dont on peut attendre la prise en charge par un automate d'analyse. Cette liste, très schématique représente un compromis. On peut se contenter de beaucoup moins ou exiger beaucoup plus selon les applications envisagées. On voit que la question particulière et cependant capitale des dosages cinétiques d'enzymes n'a pas été abordée, seuls deux ou troisconstructeurs l'ayant résolue de façon à peu près staisfaisante et la maison Technicon n'étant pas de ceux-là. (Il faut en effet que l'appareil calcule l'activité de l'enzyme à partir d'une variation supposée linéaire de la densité optique et que la portion de droite correspondante soit enregistrée en continu sur papier ou sur écran cathodique, offrant ainsi un contrôle visuel de la linéarité et de la pente de la réaction).

Tableau 2

\begin{tabular}{|c|c|}
\hline $\begin{array}{l}\text { Opérations Volumétriques } \\
\text { (sur les liquides) }\end{array}$ & $\begin{array}{l}\text { prélèvement } \\
\text { débit } \\
\text { transfert } \\
\text { dilution }\end{array}$ \\
\hline $\begin{array}{l}\text { Opêrations Mécaniques } \\
\text { (sur les liquides ou les } \\
\text { récipients) }\end{array}$ & $\begin{array}{l}\text { agitation } \\
\text { centrifugation (filtration) } \\
\text { transport }\end{array}$ \\
\hline $\begin{array}{l}\text { Opérations Thermiques } \\
\text { (sur les liquides ou les } \\
\text { récipients) }\end{array}$ & $\begin{array}{l}\text { chauffage } \\
\text { refroidissement } \\
\text { thermostatisation }\end{array}$ \\
\hline Opérations Optiques & $\begin{array}{l}\text { choix de la longueur d'onde } \\
\text { stabilisation de la source lumineuse } \\
\text { mesure photométrique, etc. }\end{array}$ \\
\hline Opérations d'Entrée/Sortie & $\begin{array}{l}\text { identification de l'échantillon } \\
\text { autocorrection (par rapport au blanc) } \\
\text { auto-étalonnage et calcul des con- } \\
\text { centrations } \\
\text { enregistrement graphique } \\
\text { digitalisation des signaux analogiques } \\
\text { affichage } \\
\text { impression } \\
\text { perforation (cartes, bandes), etc. }\end{array}$ \\
\hline Opérations Chronorrétriques & $\begin{array}{l}\text { contrôle de la succession des opérations } \\
\text { contrôle de la durée des opérations }\end{array}$ \\
\hline
\end{tabular}

Il n'est pas possible de décrire une à une toutés les solutions apportées jusqu'ici à chacun de ces problèmes, mais prenons le cas des opérations volumétriques, point critique de tous les appareils existant, -sans exception (sauf de l'AutoAnalyzer qui a eu le génie de les sup-

2) Depuis la première rédaction de ce rapport, des détails ont pu être obtenus sur ces deux instruments: - Le DSA 560 eșt capable de traiter de petits échantillons $(20 \mu l)$ à une vitesse moyenne de 150 déterminations colorimétriques à l'heure; il a l'originalité de comporter un module de filtration. - Quant à l'ACA. (Du Pont) la déception est grande. C'est une machine photométrique de conception très nouvelle mettant en oeuvre des techniques avancées mais dont le rapport prix/performances est à ce point défavorable qu'on se demande quelle clientc̀le il pourra satisfaire. Il effectue en effet une analyse à la minute, impose non seulement les réactions (souvent très discutables) mais les réactifs (spécialement conditionnés et incontrolables) et utilise un système d'identification photographique inçompatible avec un ordinateur. Son seul avantage est de pouvoir être utilisé - avec une grande souplesse - par un manoeuvre non spécialisé. C'est l'exemple même de l'appareil dont tout chimiste analyste devrait se méfier. 
primer). On peut regrouper dans quatre catégories les techniques actuellement utilisées pour mesurer les liquides. Le péristaltisme, repris de l'AutoAnalyzer, est une technique peu précise, utilisée surtout pour l'alimentation de cuves photométriques à flux continu, mais certains (comme Elvi) l'appliquent au prélèvement et à la dilution du sérum, en contrôlant électroniquement la durée d'action des galets presseurs. Les valves rapides, généralement électromagnétiques, introduites dans des conduits sous pression ou dépression constantes et dont le temps d'ouverture est contrôlé électroniquement servent aussi bien à l'alimentation de photomètres (chez Gilford, par exemple) qu'au débit de réactifs (chez Poli-Mak, par exemple). L'utilisation de pipettes jaugées, fondamentale en analyse manuelle, n'est que très peu répandue. Un système a été décrit et, peutêtre commercialisé, basé sur des pipettes à débordement mises alternativement sous vide et sous pression. A un stade beaucoup plus avancé, la pipette à jet de Guigan s'apparente à ce système (la place nous manque malheureusement pour la décrire ici). Mais c'est l'utilisation de pistons (ou de plongeurs) à mouvement alternatif qui reste la plus fréquente. Pour être fiables, ces systèmes doivent être étanches, leur mouvement doit être progressif, (idéalement: sinusoïdal) et synchronisé avec l'ouverture et la fermeture de robinets à voies multiples. Comme dans le reste du système, les liquides ne doivent entrer en contact qu'avec du verre ou des polymères fluocarbonés. Il faut se souvenir, en particulier que le Teflon est déformable et qu'un joint verre-Teflon n'est étanche, à longue échéance, qu'à condition de subir la contrainte d'un élément élastique, de préférence incorporé au piston ou au sphincter du plongeur; joint torique en élastomère (chez AutoKemi, par exemple) ou ressort d'acier (chez PolyMetron, par exemple).

En ce qui concerne la course du piston, il faut se méfier des mouvements uniformes à démarrage brusque et à butée d'arrêt réglable, et préférer les commandes précalibrées en usine, par cames, excentriques ou moteurs spéciaux, tels le moteur à impulsions ou le variateur sinusoïdal. Les valves passives, avec ou sans ressort de rappel, agissent généralement avec un retard qui dépend de la viscosité du liquide, et leur étanchéité est douteuse; il faut donc rechercher les robinets synchronisés (à boisseaụ ou à tiroir), pour lesquels les combinaisons Teflon/KelF et verre/Teflon chargé de verre sont les plus satisfaisantes. On peut signaler ici la solution élégante adoptée par Eppendorf d'un diluteur sans valves, avec, pour corollaire le léger inconvénient d'un réservoir limité de réactif.

Ces réflexions sont données à titre d'exemple, elles montrent que le chimiste peut décider de la valeur d'un instrument sur la base d'un petit nombre de critères simples. Et, la tendance étant aux instruments modulaires, cet esprit critique est payant puisqu'il permet un choix judicieux d'unités fonctionnelles qui peuvent être compatibles alors même qu'elles proviennent de constructeurs différents.

\section{Analyse Rapide}

L'un de ces critères, celui des possibilités de contamination d'un échantillon à l'autre, nous amène à distinguer deux tendances bien différentes. En effet, si on néglige l'extrémité de la pipette à prélèvement, qui est généralement abondamment rincée par le premier réactif, le seul point de rencontre des échantillons se situe à l'extrémité de la chaîne, dans la cuve du photomètre. Dans la règle, l'analyse discontinue s'achève en analyse continue. Les cycles de remplissage-lecturevidange-rinçage limitent la rapidité et la sensibilité des méthodes; les plus courts sont de quinze secondes et ils requièrent un volume final de solution hors de proportion avec les possibilités des micropipettes. Certains constructeurs (comme PoliMak) ont donc voulu faire "mieux que la laborantine idéale», en effectuant les réactions dans les cuvettes photométriques elles-mêmes. La solution la plus élaborée dans ce sens est présentée par la Maison LKB, dont le module photométrique exécute 1.500 lectures à l'heure dans des cuvettes perdues, valeurs calculées et imprimées.

Nous voici amenés au coeur du problème: faut-il donner la priorité à l'automatique ou à la rapidité?

Compte tenu du fait qu'un appareil est généralement d'autant plus lent, plus compliqué, plus cher, et que son entretien requiert un personnel d'autant plus spécialisé qu'il est automatisé, il vaut beaucoup mieux accélérer les analyses cliniques à l'aide d'appareils semi-automatiques rapides, de maniement simple, sur lesquels il est facile de garder le contrôle et dont les défaillances n'immobilisent pas toute une chaîne de travail. Il n'est pas urgent d'automatiser le chauffage et la thermostatisation de cent éprouvettes, puisqu'il suffit pour cela de porter un plateau dans une étuve, mais il serait précieux de pouvoir se dispenser d'en mesurer le contenu deux à deux dans les cuvettes ultramicro d'un photomètre à double faisceau, ce qui représente déjà pourtant la situation d'un laboratoire privilégié. Il faut utiliser des microméthodes "directes», en rationaliser et en optimaliser les étapes successives et surtout, commencer l'automatisation des chaînes d'analyse "par la fin" c'est à dire par la mesure.

Nous ne dirons rien de la pratique qui consiste à associer en parallèle des chaînes d'analyse complètes et entièrement automatiques, pour obtenir des ensembles multicanaux aux performances impressionnantes, puisqu'ils exécutent (ou se proposent d'exécuter) trois mille dosages à l'heure (24/135 échantillons chez AutoKemi; $10 / 300$ échantillons chez Vickers et Elvi). Après l'erreur du SMA 12/30, sur laquelle nous glisserons pudiquement, l'AutoAnalyzer s'est lui aussi. mis à la page, avec le SMA 12/60, où un certain nombre des faiblesses du système originel ont été ingénieusement -surmontées, mais cette machine très perfectionnée n'exécute que 680 dosages à l'heure, dont plus de la moitié n'ont pas de valeur diagnostique. Elle conviendrait pour un petit hôpital très riche. 
En réalité, il n'y a guère de limites à ce genrc d'addition. Nous pensons surtout que ces machines rigides et coûteuses viennent trop tôt, que ni les médecins ni les chimistes n'ont mérité cela. La chimie clinique est encore trop jeune, elle doit encore se simplifier et s'améliorer considérablement. Ainsi, nous n'avons rien contre la photométrie d'absorption dans le visible, mais il ne faudrait pas que cette technique fasse oublier l'ultraviolet, la fluorimétrie, l'absorption atomique, l'électroanalyse, la chromatographie et les différentes formes de spectrométrie d'émission.

(Un coulomètre ou un photomètre de flamme, ou d'absorption atomique, peuvent afficher instantanément un résultat digital calculé, sur simple présentation de l'échantillon brut ...).

Quant à la photométrie classique, on peut faire mieux: un seul module du système Guigan, fabriqué actuellement par la Compagnie générale d'Automatisme, exécute des analyses complètes au rythme de deux par seconde, et pour un prix dérisoire, préfigurant ainsi ce que sera la chimie clinique de demain, car d'autres constructeurs ont compris le rôle primordial de la rapidité. Mais tous ces efforts manquent encore de coordination. Les médecins, les biochimistes et les analystes doivent préparer cette chimie clinique nouvelle et engager le dialogue avec l'industrie, de manière à obtenir les meilleurs instruments possible. La conversion analogique/digitale, par exemple, est une des fonctions qui doit être assurée par le photomètre luimême; le problème des solutions corrosives ou visqueuses est à résoudre par le chimiste. Le rôle des ordinateurs n'est pas, comme on le croit trop souvent, de surveiller et de corriger des instruments imparfaits, pas plus que celui des constructeurs n'est de mécaniser des méthodes périmées et inutilement compliquées. Il semble bien que, dans l'état actuel des choses, la contri- bution des chimistes soit de celles qui font le plus défaut.

En attendant cette deuxième génération d'automates, que les systèmes automatiques continus ou non, trop lents pour la plupart, cèdent la place à l'analyse manuelle accélérée. Cela est moins facile mais beaucoup plus fécond et, à long terme, rentable.

\section{Abstract \\ Automatic continuous and discontinuous analysis and rapid analysis}

Between the era of the AutoAnalyzers, pioneers in automated clinical chemistry, and the supermachines of tomorrow, able to make several tens of thousands determinations per hour, the analyst in 1968 finds his attention drawn to numerous instruments which have not yet achieved the level of speed necessary for efficiency. Slow mechanization is often worse than no mechanization at all. This fundamental weakness together with a certain number of others which, being less basic, can be more easily overcome, allows us to group these instruments into a premature generation of mechanized equipment.

The analyst, being aware of these deficiencies, must not be content mercly to denounce the imperfections and limits of the products offered to him on the market, but rather should actively participate in their elaboration through improved methods, and by putting forward detailed recommendations on the most suitable way to mechanize them.

The analysis systems must produce digitized and readily intelligible data without the assistance of computers, that is, they must correct themselves rather than be monitored by data processing devices. The need for flexibility, security and efficiency makes the interpenetration (i. e. the interdependance) of these two categories of instruments undesirable.

It is by co-ordinating the specialist knowledge and responsibility of biochemists, analysts, instrument manufacturers and computers programmers that clinical chemistry will quickly emerge from the crisis into which it is sinking.

The mechanization derices at pressent available constitute intermediary materials to be used with caution. It must be ensured in the first place that they will not impair analytical methods and that they will work at least as fast as a trained technician.

\section{References}

1. Skeggs, L. T., Amer. J. Clin. Path. 28,311 (1957). - 2. Thiers, - 4. Scheuerbrandt, G. et R. Helger, in press. - 5. Härter, R. E., R. R. Cole et W. J. Krrsch, Clin. Chem. New York 13, 451 (1967). - 3. Hughes, H. K., Analytic Chem. 24, 1349 (1952).

A., R. Helger et H. LANG, Ce journal 7, 14 (1969).

Dr. J. Bierens de Haan Hôpital Cantonal CH 1211 Geneve 4 


\section{SH A N D O N}

\section{Disc- \\ Elektrophorese auf Polyacrylamidgel}

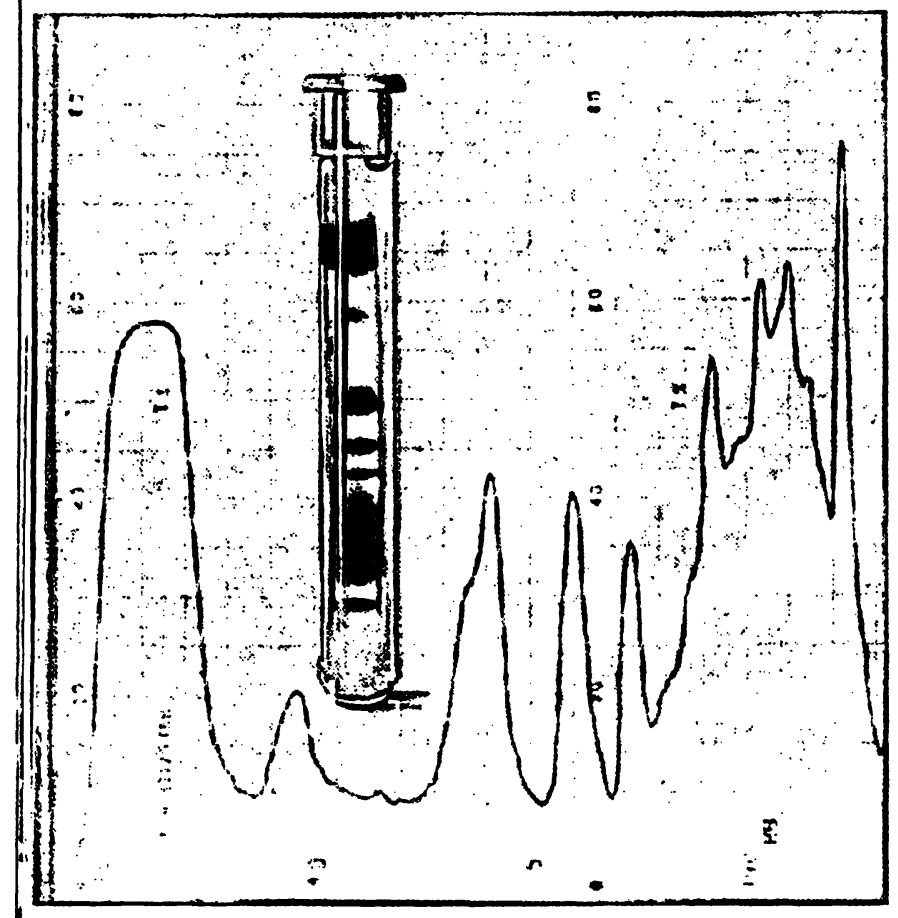

Die Sãulenelektrophorese auf Polyacrylamidgel (Disc-Elektrophorese) bietet der Forschung weitere Mőglichkeiten. Die Fraktionen nehmen die Form scharf getrennter, schmaler Scheiben an. Häufig können 25 bis 30 Serumfraktionen im Molekulargewichtsbereich von 10000 bis 1 Million erhalten werden.

Die Disc-Elektrophorese bietet hohe Auflösungen bei Serum, Hämoglobin, Enzymen, Glyco- und Muco-Proteinen, Milch, Peptonen, Spinalextrakten, Ribonukleinsäuren, Zellextrakten u. a.

Wir liefern komplette Aușūstungen für analytische und präparative Arbeiten.

SHANDON LABORTECHNIK GMBH 6 FRANKFURT/MAIN 50 - Karl von Drais-Straße 18 Postfach 29 . Telefon (0611) 541065

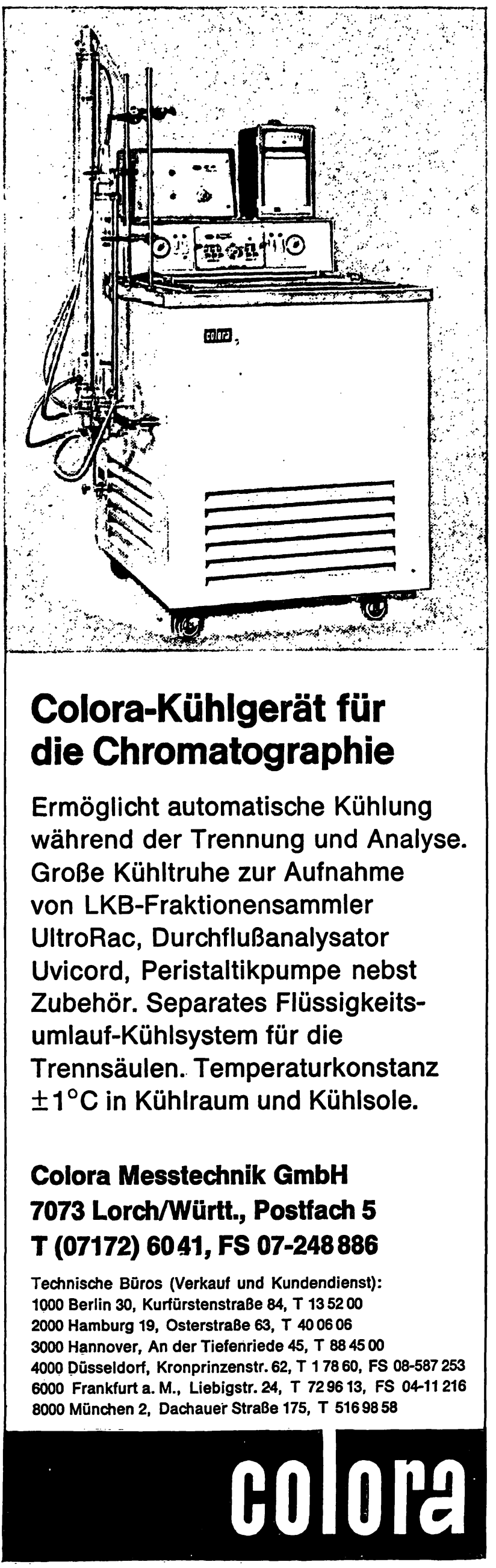




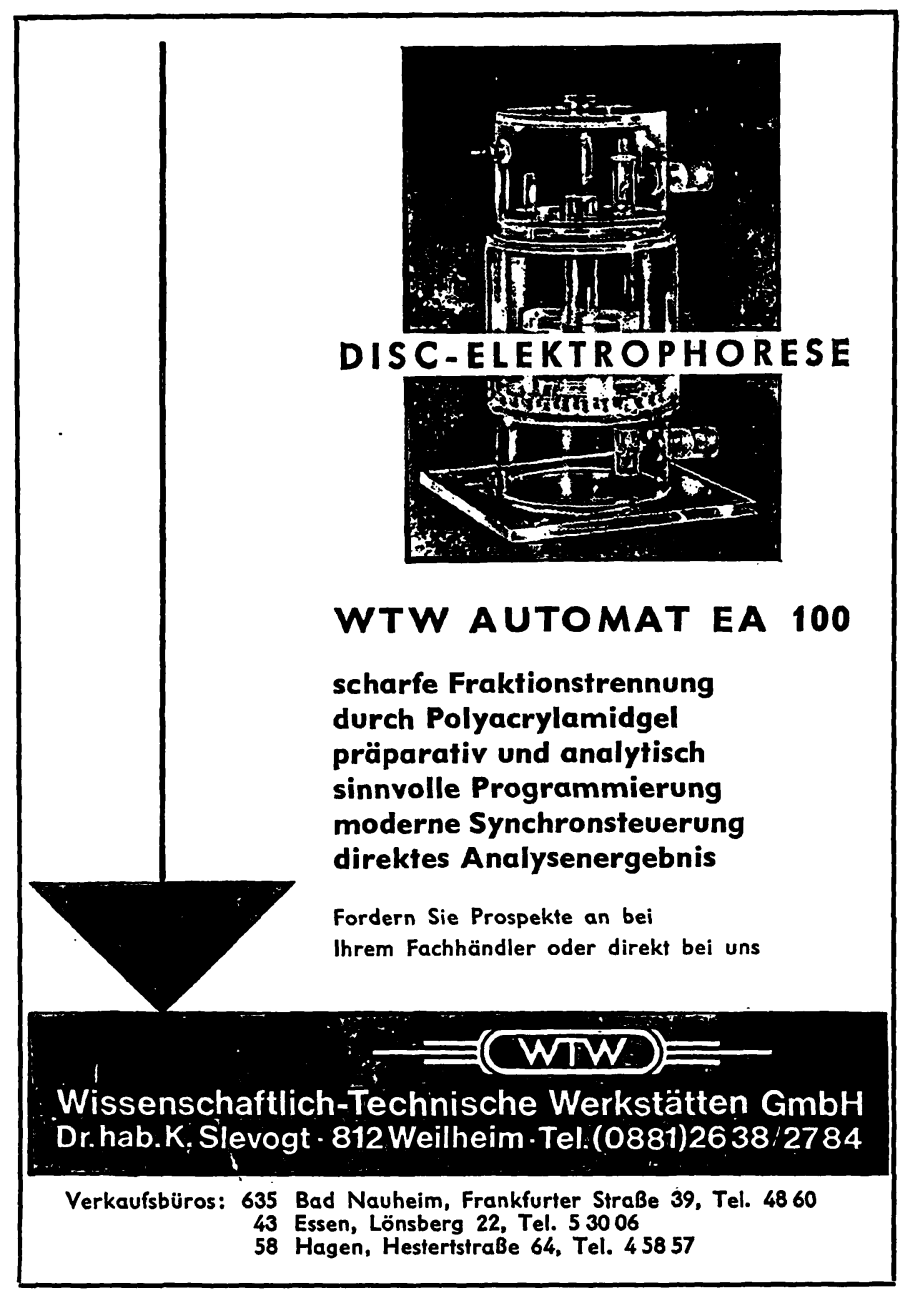

\section{Die Injektion}

\section{Technik - Praxis - Komplikationen}

Von Priv.-Doz. Dr. med. Dr. med. dent. Joachim Gabka

Groß-Oktav. VII, 205 Seiten. Mit 73 z. T. mehrfarbigen Abbildungen. 1968. Plastik flexibel DM 20,-

Es ist dringend an der Zeit, der Fachwelt ein Werk vorzulegen, in dem sowohl Technik als Fehler sowie Gefahren der Injektionsbehandlung aufgezeigt werden. - Jeder Student der Medizin, jeder Medizinalpraktikant, jeder junge Mediziner, jedoch auch die erfahrenen Ärzte sollen durch die Darlegung der Komplikationsmöglichkeiten bei der Injektionstechnik sowie durch die in der Literatur beschriebenen Zwischenfälle orientiert werden. Der Autor hat die einzelnen Injektionsarten und deren Komplikationsmöglichkeiten aufgezeigt sowie die Therapie dieser Vorkommnisse dargelegt. - Mit besonderer Sorge, aber auch mit Recht weist der Autor daraufhin, daß in letzter Zeit die Deutschen Gerichte bei Schadensersatzprozessen als Folge von Injektionsschäden kaum noch den sachlichen Darlegungen des zugezogenen Gutachters folgen und die beklagten Årzte zumeist wegen Fahrlässigkeit verurteilen.

Walter de Gruyter \& Co. Berlin 30

\section{Pherograph}

\section{Original Frankfurt} nach

\section{Wieland-Pfleiderer}

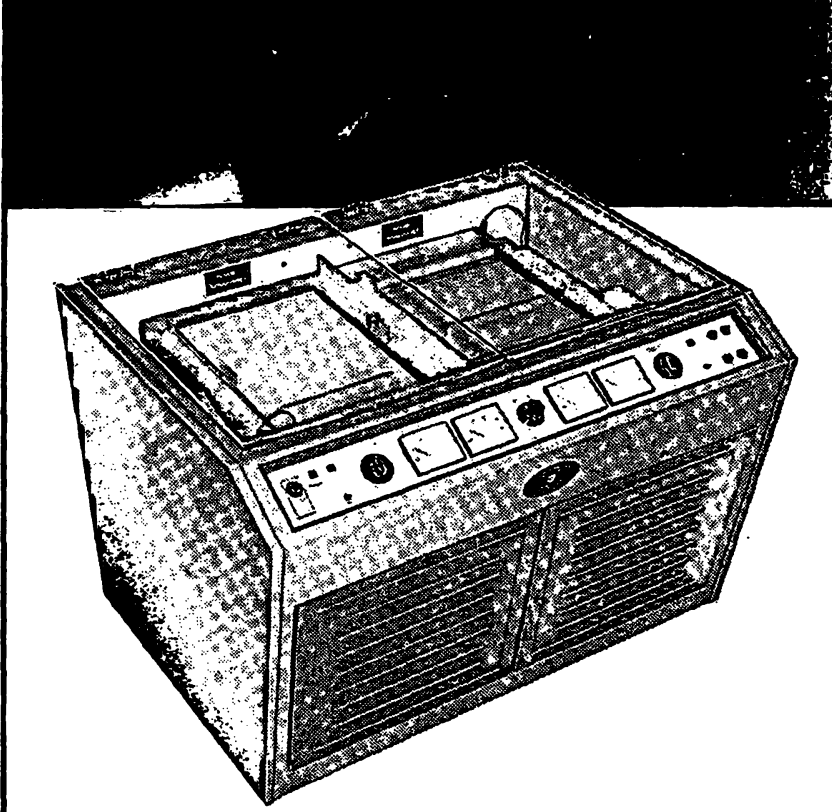

Geräte für die analytische und präparátive Hoch-und Niederspannungselektrophorese Ein- und Zweikammersystem, gekühlt, komplett mit Spannungsvervielfacher.

\section{Anwendungsgebiete:}

Analytische, organische, anorganische, physikalische Chemie; Biochemie, Pharmakologie, Medizin.

Trennung von geladenen Partikeln, z. B. anorganischen Kationen und Anionen, organischen Säuren und Basen, Aminosäuren, Peptiden, Nucleotiden, Zuckern u. a.

Mikropräparative Trennung von Proteinen in Trägerschichten wie Stärke, Cellulosepulver usw.

\section{Mikro-Imun Elektrophorese}

Elektrophoretische Entsalzung

Isotopentechnik

Dünnschichtelektrophorese

HOR MUTH - VETTER

6900 Heidelberg 1, Postf. 750, Tel. 06221/20045 6908 Wiesloch/Bd., Postf. 1145, Tel. 06222/2147 